\title{
Denervated muscle fibres in hereditary mouse dystrophy
}

\author{
A. J. MCCOMAS AND K. MROŻEK \\ From the Physiology Department, University of Newcastle upon Tyne, and Muscular Dystrophy \\ Laboratories, Newcastle General Hospital
}

Muscular dystrophy in the mouse, as in man, is considered to be a genetically determined primary myopathy in which abnormalities of motor innervation are secondary to degeneration of muscle fibres (cf. Harman, Tassoni, Curtis, and Hollinshead, 1963). In the mouse, however, there is now evidence to indicate that the motor nerve supply to a fibre may be affected at a stage in the disease when the fibre itself is still viable. Thus dystrophic fibres in sufficiently good condition to have resting potentials of 60 to $80 \mathrm{mV}$ may receive abnormally small quanta of acetylcholine from motor nerve terminals (McComas and Mossawy, 1965); similarly Conrad and Glaser (1964) have demonstrated that the rate of spontaneous quantal emission may also be reduced.

In the present study we have explored the possibility that for certain of the dystrophic fibres a more extreme situation may exist such that part, or all, of a fibre is completely free from nervous control. Our results suggest that such a condition is surprisingly common in dystrophy and, furthermore, that it may affect fibres which have electrophysiological evidence of viability. The implications of this finding are felt to be of some significance in relation to the features of the disease.

\section{METHODS}

PREPARATION The experiments were conducted on eight dystrophic mice of the Bar Harbor 129 strain and on 15 control mice, of which nine were homozygous or heterozygous litter mates of the affected animals while six belonged to the C.B.A. strain. The ages of the mice ranged from 6 to 14 weeks; all the dystrophic animals had evidence of weakness and wasting of skeletal muscles. The mice were anaesthetized by intraperitoneal injection of a $25 \%$ urethane solution $(2 \cdot 0-2 \cdot 5 \mathrm{~g} . / \mathrm{kg}$. body weight) and after a tracheostomy had been performed they were mounted so that the lower half of the body was enclosed in a Perspex bath; the bath was filled with a mammalian bathing fluid (Liley, 1956) maintained at approximately $33^{\circ} \mathrm{C}$. In some experiments the tibialis anterior muscle was studied while in others the gastrocnemius was explored following a careful dissection of the overlying biceps femoris. The sciatic nerve was cut in the upper thigh and the distal end was placed over a pair of silver wire stimulating electrodes which were immersed in the bathing fluid; no attempt was made to dissect the branches of the sciatic nerve.

The time and the amount of dissection required in the present experiments were considerably greater than those of an earlier investigation which was largely concerned with resting potential amplitudes (McComas and Mossawy, 1965). Both these factors may have been responsible for a slight deterioration in the condition of the muscle fibres sufficient to account for the rather lower resting potentials found in the present study (see Results).

STIMULATION AND RECORDING The arrangement used for directly stimulating, and for recording from, single muscle fibres with $\mathrm{KCl}$-filled microelectrodes connected through a Wheatstone bridge has been described previously (McComas and 'Mossawy, 1966; cf. Beránek, 1964). In the present study the penetration of a fibre was followed by direct stimulation using the technique of 'break' excitation described by Beránek (1964). This method had the advantage that it could be used for microelectrodes with high resistances since it did not matter if the electrode rectified during the passage of the 30 msec. cathodal current. In a small proportion of fibres the threshold to direct stimulation was greater than the maximum current of the stimulating system $\left(1 \cdot 1 \times 10^{-6} \mathrm{~A}\right)$. For the remaining fibres $(96 \%$ of the normal and $93 \%$ of the dystrophic populations respectively) the thresholds ranged from $0.8 \times 10^{-7} \mathrm{~A}$ to $11.5 \times 10^{-7} \mathrm{~A}$ with a mean of approximately $6 \times 10^{-7} \mathrm{~A}$. This mean value was approximately ten times as large as that of the threshold for intracellular anodal stimulation (McComas and Mossawy, 1966).

The initiation of an action potential upon direct stimulation was regarded as critical evidence that the microelectrode tip was inside a viable muscle fibre; the sciatic nerve was then stimulated and indirect excitation of the fibre looked for. The stimulus to the nerve was 0.3-0.5 msec. in duration and was made supramaximal for motor nerve fibres. In early experiments this was achieved by carefully observing the amplitudes of the muscle twitches under a dissecting microscope as the stimulus strength was gradually increased; when the twitches appeared to be maximal the stimulating voltage 
was doubled. In later experiments proof that the stimulus was supramaximal was obtained by monitoring the electromyogram on the surface of the muscle with a pair of $0.25 \mathrm{~mm}$. diameter silver wires insulated to their tips with polythene. If a fibre failed to be excited indirectly the direct stimulation was repeated since it was essential to verify that the fibre had not been damaged or dislodged from the microelectrode during the previous twitches. Each response, whether to direct or to indirect stimulation, was photographed for subsequent analysis. The position of every fibre investigated was noted on an enlarged freehand drawing of the muscle.

STATISTICAL TREATMENT OF RESUlts Mean values in the text and in the illustrations have been presented with the standard errors of the means. Significances of differences between means have been calculated using the $t$ test.
RESULTS

INCIDENCE OF DENERVATION The present investigation differs in two important respects from the earlier one of McComas and Mossawy (1965) who were unable to obtain convincing evidence of denervation in dystrophic tibialis anterior muscles. First, intracellular stimulation has been used to establish conclusively whether or not the electrode tip lay inside a viable muscle fibre. This evidence was crucial since otherwise the failure to evoke an action potential on indirect stimulation could have been mistakenly interpreted as evidence that the fibre was damaged, or alternatively that the electrode tip was not inside a fibre at all but merely
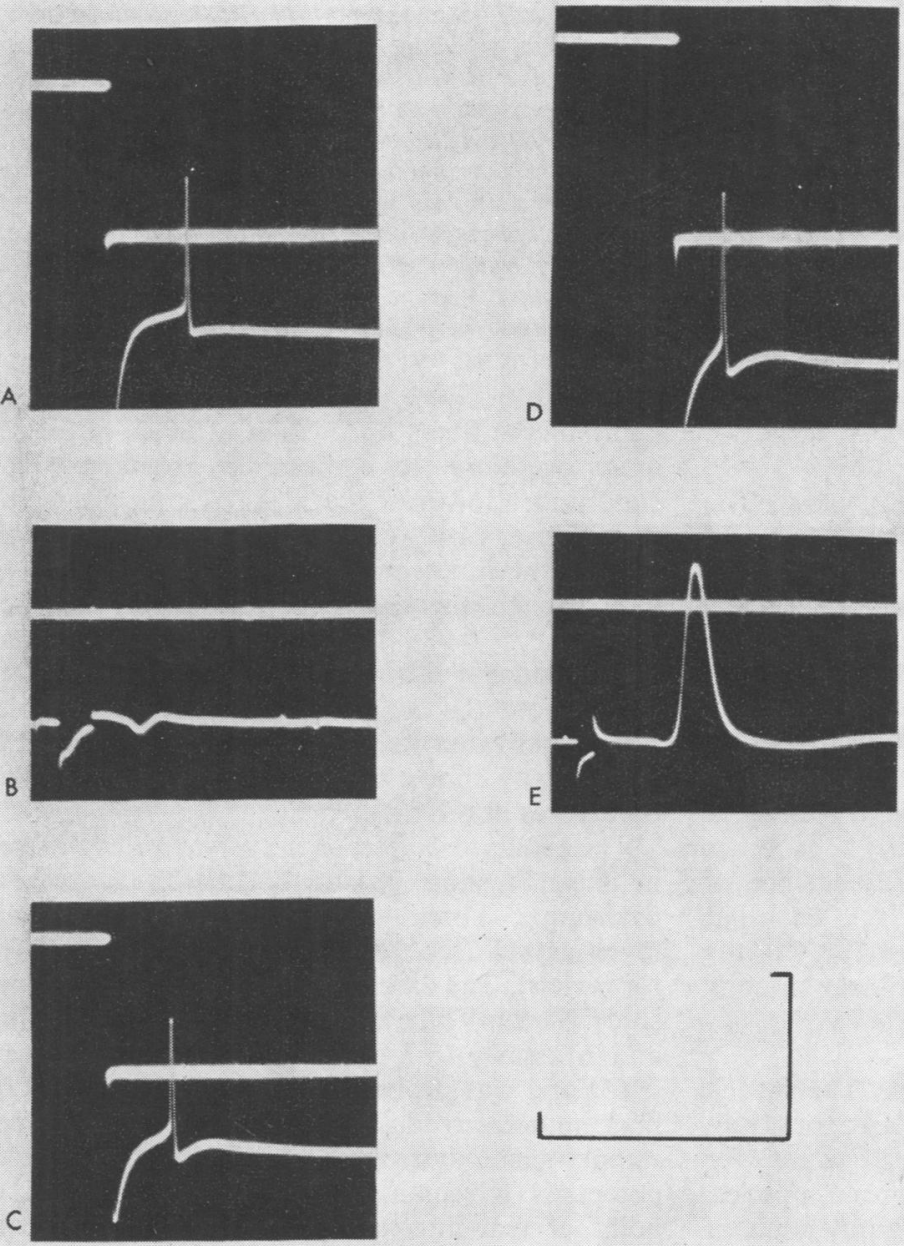

FIG. 1. Intracellular recordings from a dystrophic muscle fibre $(A, B, C)$ and a control fibre $(D, E)$ to demonstrate denervation in the former. $A, C$, $D$ are photographs of intracellular cathodal stimulation (spikes retouched). The termination of the rectangular current pulse is recorded on the top trace of each photograph and its amplitude is indicated by the vertical calibration mark (lower right) representing $6 \times 10^{-7} \mathrm{~A}$. The intracellular responses are on the lower trace and in each case action potentials follow termination of the current pulses, i.e., 'break' excitation has occurred; for these traces the vertical calibration represents $100 \mathrm{mV}$ and the horizontal one 50 msec.; the baseline of the upper trace approximates to zero membrane potential. $B$ and $E$ are responses to nerve stimulation; vertical and horizontal calibrations are $100 \mathrm{mV}$ and 5 msec. respectively.

The dystrophic fibre was regarded as denervated since it responded to intracellular 'break' stimulation $(A)$, but not to nerve stimulation $(B) ; a$ further 'break' excitation (C) indicated that the microelectrode tip was still inside the muscle fibre. The control fibre responded both to 'break' stimulation (D) and to nerve stimulation $(E)$; it was therefore innervated. 
recording a negativity resulting from a change in tip potential or from the penetration of some other structure. Secondly, in the present study we have employed rather finer microelectrodes, with resistances of approximately $20 \mathrm{M} \Omega$, so as to record more easily from the relatively small fibres in dystrophic muscles.

A fibre was regarded as 'denervated' if it responded to direct but not to indirect stimulation and an example is shown in Figure 1, together with the responses of an innervated fibre for comparison. Altogether 181 dystrophic fibres were investigated and of these no fewer than $48(27 \%)$ were judged to be denervated. Using the same criteria five of 208 fibres in litter mate controls appeared to be denervated. Since the genotype of the litter mate controls was not known it was conceivable that the denervated fibres in these animals could have been an expression of subclinical dystrophy in heterozygous carriers. Thus it is now established in man that carriers of Duchenne dystrophy may have electrophysiological, biochemical, and histological evidence of muscle involvement (cf. Walton and Pennington, 1966). A further set of experiments was therefore conducted on six mice of the C.B.A. strain, a strain which is not susceptible to hereditary muscular dystrophy. In a total of 219 fibres, no evidence of denervation was found. Therefore, since there was no reason to question the reliability of the experimental method, it seems at least possible that a small amount of denervation may have been a feature of heterozygous animals.

DISTRIBUTION OF THE DENERVATED FIBRES The incidence of denervated fibres in dystrophic muscles varied widely and did not show any correlation with the apparent weakness of the hindlimbs. Thus in one animal with a complete flaccid paraplegia only $9 \%$ of fibres were denervated while in another animal, which could still use the hindlimbs for walking, $46 \%$ of the fibres were affected. The distribution of the denervated fibres within a muscle also varied. In some muscles there was a focal area which did not appear to twitch as well as other parts of the muscle and in which the incidence of denervation was very high (Fig. 2, A). In other muscles, such as the tibialis anterior in Fig. 2B, the denervated fibres were scattered diffusely among innervated ones.

RESTING POTENTIALS OF MUSCLE FIBRES The resting potentials of the denervated fibres are shown in Fig. 3 together with corresponding values for innervated fibres in dystrophic and normal muscles. It was found that although the resting potentials of the denervated dystrophic fibres varied considerably
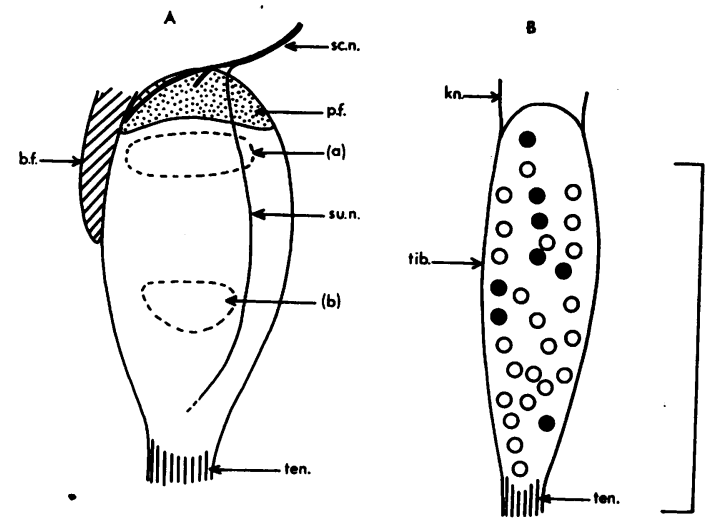

FIG. 2. Distribution of denervated muscle fibres in two dystrophic muscles. $A$ is a gastrocnemius muscle which showed focal denervation. Two zones of the muscle were explored and their extents are indicated by interrupted lines. In the proximal zone (a) only one of 11 fibres was denervated while in in the distal zone (b) six of eight fibres were denervated; furthermore, the latter region did not appear to twitch well on microscopic examination.

$B$ is a tibialis anterior muscle in which the superficial fibres were uniformly sampled (as in all muscles other than A). In this muscle the denervated fibres (filled circles) had an apparently random distribution amongst innervated ones (open circles). Vertical scale, $10 \mathrm{~mm}$. Sc.n., sciatic nerve; p.f., popliteal fat pad; b.f., biceps femoris muscle (cut); su.n., sural nerve; ten, tendon; kn., knee; tib., tibia.

many of the fibres had values of 60 to $80 \mathrm{mV}$ and must therefore have been in reasonably good condition. However, the mean resting potential of these fibres, $61.6 \pm 1.52 \mathrm{mV}$, was rather lower than that of the innervated dystrophic ones, $65 \cdot 1 \pm 0 \cdot 67$ $\mathrm{mV}$, though the difference was barely significant $(0.05>P>0.02)$. Nevertheless the result was of interest since it is now well established that a fall in resting potential occurs in normal fibres which have been denervated and, in addition, Lenman (1965) has recently shown that this is also true of dystrophic fibres in the mouse.

The results also showed the very marked difference in mean resting potential between normal and dystrophic fibres already reported by several investigators (see McComas and Mossawy, 1965, for review). The difference was greater in the present study $(11.9 \mathrm{mV})$ than in the earlier one of McComas and Mossawy (6.7 mV; McComas and Mossawy, 1965 ) and was probably due to the introduction of finer microelectrodes which would be better able to sample small dystrophic fibres having low resting potentials (Kleeman, Partridge, and Glaser, 1961). A point of real interest, however, was that the low mean resting potential of dystrophic fibres was not 

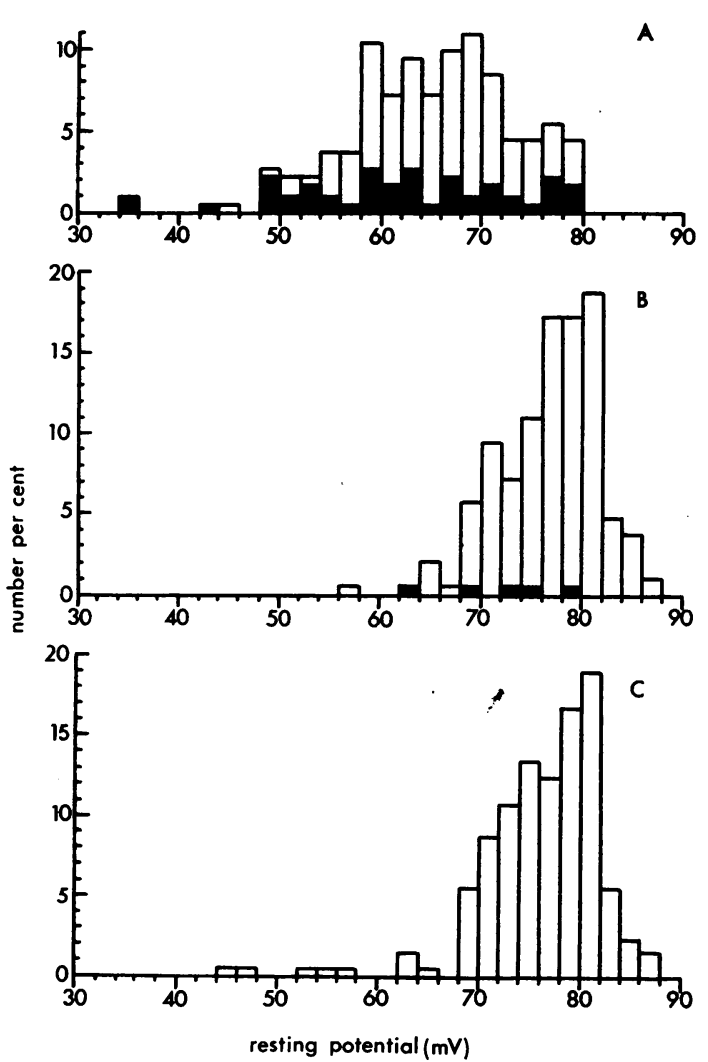

FIG. 3. Resting potentials of denervated and innervated muscle fibres (filled and open columns respectively).

$A, 181$ fibres from dystrophic muscles, overall mean $64.2 \pm 0.62 \mathrm{mV}$; denervated mean $61.6 \pm 1.52 \mathrm{mV}, n=48$; innervated mean $65 \cdot 1 \pm 0.67 \mathrm{mV}, n=133$.

$B, 208$ fibres from littermate controls, overall mean $76 \cdot 1 \pm 0.29 \mathrm{mV}$; denervated mean $70 \cdot 8 \pm 3 \cdot 20 \mathrm{mV}, n=5$; innervated mean $76 \cdot 2 \pm 0 \cdot 29, n=203$.

C, 219 fibres from C.B.A. controls, mean $75 \cdot 8 \pm 0 \cdot 38 \mathrm{mV}$.

due to the inclusion of denervated fibres since the mean value for innervated dystrophic fibres, $65 \cdot 1 \pm 0.67 \mathrm{mV}$, was also significantly reduced $(\mathbf{P}=<0.001)$.

\section{DISCUSSION}

The results of the present investigation indicate that denervation is a common occurrence in dystrophic mouse muscles and must therefore be considered as a major factor contributing to muscular weakness. On the basis of histological and electophysiological studies it seems quite likely that denervation may take place in the vicinity of the neuromuscular junction. Thus Curtis, Abrams, and Harman (1961) have reported such changes as elongation and fragmentation of endplates and both McComas and Mossawy (1965) and Conrad and Glaser (1964) have found electrophysiological evidence of neuromuscular dysfunction (see Introduction). Another potential site for the onset of denervation should also be considered in dystrophy; it seems possible that a necrotic segment within a fibre could cause functional denervation by interrupting the spread of electrical and trophic activity from the endplate. If the first hypothesis is correct the whole of the fibre should be denervated (Fig. 4, A); by com-

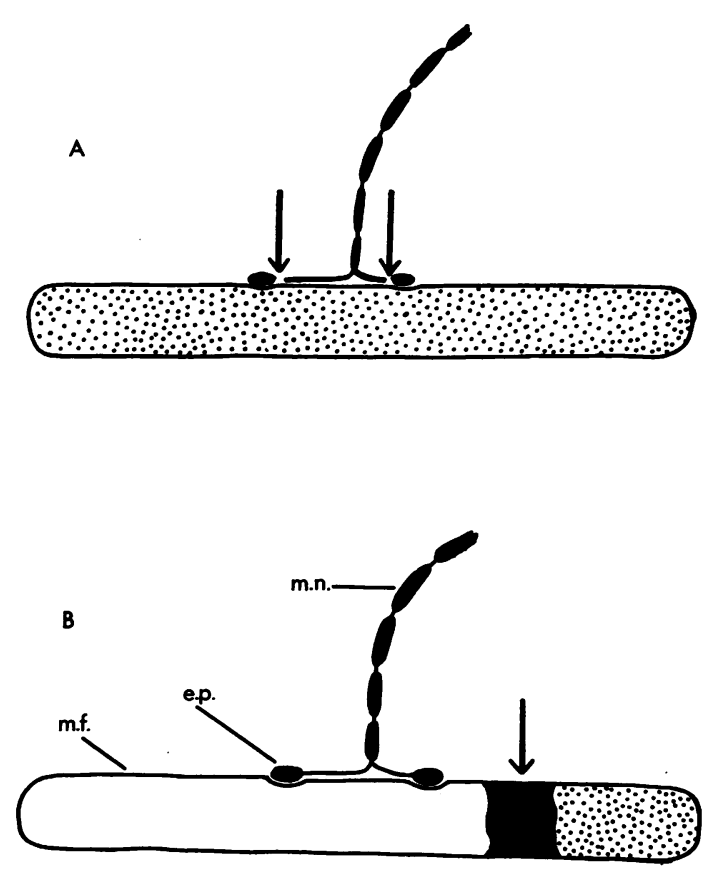

FIG. 4. Two hypothetical schemes to account for denervation (see text). In $A$ the arrows indicate a lesion at the neuromuscular junction resulting in denervation of the complete muscle fibre. In B, a necrotic segment (black area, arrowed) within a fibre causes functional denervation of part of fibre only.

Dotted areas indicate extent of denervation; m.n., motor nerve axon; e.p., endplate; m.f. muscle fibre.

parison the second hypothesis predicts that only the part of the fibre distal to the necrotic lesion will be affected (Fig. 4, B) but that a larger proportion of fibres will be involved.

In fact the true incidence of denervation could actually be higher than either hypothesis would suggest since neither would include those denervated fibres which had deteriorated so far as to be inexcitable. In the present study, however, the ampli- 
tudes of the resting potentials, together with the evidence of excitability on direct stimulation, both indicate that denervated fibres may be in reasonably good condition. It follows that the morphological appearance of a dystrophic muscle may give a quite misleading impression as to its mechanical properties since a proportion of the fibres, although apparently viable and containing myofibrils, may be functionally useless.

The final point to emerge from this study is that denervation should be taken into account as a possible factor contributing to the histological, biochemical, and electrophysiological features of muscular dystrophy. So far as electrophysiological abnormalities are concerned the effect of denervation would have to be considered in relation to fibrillation potentials (Mossawy, 1966), reduction in membrane potentials (cf. McComas and Mossawy, 1965, for review), and changes in membrane conductance (McComas and Mossawy, 1966). Histologically it is well known that a reduction in fibre size and the presence of chains of central nuclei are features of denervation (cf. Gutmann and Zelená, 1962) as well as of dystrophy. Indeed Curtis et al. (1961) have made the intriguing observation that 'no evidence of innervation has yet been found in the characteristic dystrophic fibres with central nuclei'.

At present there is no evidence as to whether denervation may occur in human forms of dystrophy. If, however, the lesion producing denervation is situated in the region of the neuromuscular junction it may prove relevant that certain similarities in the innervation pattern have been reported between mouse dystrophy on the one hand and dystrophia myotonica and Duchenne dystrophy on the other (cf. Harman et al. 1963). Conversely, if the main factor producing denervation is focal necrosis of fibres (see above) then one would again anticipate that both types of human dystrophy might be affected.

\section{SUMMARY}

An electrophysiological technique has been employed to demonstrate the common occurrence of denervated muscle fibres in hereditary mouse dystrophy.

The distribution of the denervated fibres within the dystrophic muscles and the resting potentials of the affected fibres have been studied.

The results of this investigation have been assessed in terms of the morphological and electrophysiological features of dystrophic muscles.

This investigation was carried out while one of us, K.M., was in receipt of grants from the Medical Research Council of Great Britain, the Muscular, Dystrophy Group of Great Britain, and the Muscular Dystrophy Associations of America, Inc. We are indebted to Mr. W. H. Stanton and other members of the technical staff for valuable assistance.

\section{REFERENCES}

Beránek, R. (1964). Intracellular stimulation myography in man. Electroen. clin. Neurophysiol., 16, 301-304.

Conrad, J. T., and Glaser, G. H. (1964). Spontaneous activity at myoneural junction in dystrophic mice. Arch. Neurol. (Chic.), 11, 310-316.

Curtis, R. L., Abrams, M. T., and Harman, P. J. (1961). The myoneural junction in dystrophic and atrophic mouse muscle. Anat. Rec., 139, 219.

Gutmann, E., and Zelená, J. (1962). Morphological changes in the denervated muscle. In The Denervated Muscle, edited by $\mathbf{E}$. Gutmann, pp. 57-102. Czechoslovak Academy of Sciences, Prague.

Harman, P. J., Tassoni, J. P., Curtis, R. L., and Hollinshead, M. B. (1963). Muscular dystrophy in the mouse. In Muscular Dystrophy in Man and Animals, edited by G. H. Bourne, and M. N. Golarz, pp. 407-456, Karger, New York.

Kleeman, F. J., Partridge, L. D., and Glaser, G. H. (1961). Resting potential and muscle fiber size in hereditary mouse muscle dystrophy. Amer. J. phys. Med., 40, 219-224.

Lenman, J.A.R. (1965). Effect of denervation on the resting membrane potential of healthy and dystrophic muscle. J. Neurol. Neurosurg. Psychiat., 28, 525-528.

Liley, A. W. (1956). An investigation of spontaneous activity at the neuromuscular junction of the rat. J. Physiol. (Lond.), 132, 650-666.

McComas, A. J., and Mossawy, S. J. (1965). Electrophysiological investigation of normal and dystrophic muscles in mice. In Research in Muscular Dystrophy. Proc. Third Symposium of the Muscular Dystrophy Group, pp. 317-341. Pitman Medical Publishing Company, London.

- - (1966). Excitability of muscle fibre membranes in dystrophic mice. J. Neurol. Neurosurg. Psychiat., 29, 440-445.

Mossawy, S. J. (1966). An electrophysiological study of dystrophic muscle in the mouse. Ph.D. Thesis, University of Newcastle upon Tyne.

Walton, J. N., and Pennington, R. J. T. (1966). Studies on human muscular dystrophy with particular reference to methods of carrier detection. Ann. N. Y. Acad. Sci., 138, Art. 1, 315-328. 